A. Langer and T. Zink

Nagoya Math. J.

Vol. 179 (2005), 1-16

\title{
GAUSS-MANIN CONNECTION VIA WITT-DIFFERENTIALS
}

\author{
ANDREAS LANGER AND THOMAS ZINK
}

\begin{abstract}
Let $X / R$ be a smooth scheme over a ring $R$. Consider the category of locally free crystals of finite rank on the situs $\operatorname{Crys}\left(X / W_{t}(R)\right)$. We show that it is equivalent to the category of locally free $W_{t}\left(\mathcal{O}_{X}\right)$-modules of finite rank endowed with a nilpotent, integrable de Rham-Witt connection. In the case where $R$ is a perfect field this was shown by Etesse [E] and Bloch [Bl]. We use the result for a construction of the Gauß-Manin connection as a de Rham-Witt connection.
\end{abstract}

We generalize ideas of Bloch [Bl] about de Rham-Witt connections to a relative situation, and apply this to construct the Gauß-Manin connection in crystalline cohomology using Witt differentials.

Let $S$ be a scheme such that $p$ is nilpotent on $S$. Let $X$ be a scheme over $S$. Then the Witt differentials $W_{t} \Omega_{X / S}^{1}$ are defined in [L-Z] for each natural number $t$. These sheaves on $X$ satisfy the same exact sequences and functorialities as the usual Kähler differentials: Let $X \rightarrow Y \rightarrow S$ be morphisms of schemes. Then there is an exact sequence of sheaves on $X$.

$$
W_{t}\left(\mathcal{O}_{X}\right) \otimes_{W_{t}\left(\mathcal{O}_{Y}\right)} W_{t} \Omega_{Y / S}^{1} \longrightarrow W_{t} \Omega_{X / S}^{1} \longrightarrow W_{t} \Omega_{X / Y}^{1} \longrightarrow 0 .
$$

The reader will deduce this easily from the isomorphism (4.1).

Assume moreover that $X \rightarrow Y$ is a closed immersion defined by a sheaf of ideals $\mathfrak{a} \subset \mathcal{O}_{Y}$. One can proof in the same way that there is an exact sequence:

$$
W_{t}(\mathfrak{a}) / W_{t}(\mathfrak{a})^{2} \stackrel{d}{\longrightarrow} W_{t}\left(\mathcal{O}_{X}\right) \otimes_{W_{t}\left(\mathcal{O}_{Y}\right)} W_{t} \Omega_{Y / S}^{1} \longrightarrow W_{t} \Omega_{X / S}^{1} \longrightarrow 0 .
$$

The structure of $W \Omega_{X / S}^{1}$ in the case where $X$ is an affine space over $S$ is determined in [L-Z, Proposition 2.17].

Let $E$ be a locally free $W_{t}\left(\mathcal{O}_{X}\right)$ module on $X$. A de Rham-Witt connection on $E$ is a homomorphism of sheaves of abelian groups on $X$

$$
\nabla: E \longrightarrow W_{t} \Omega_{X / S}^{1} \otimes_{W_{t}\left(\mathcal{O}_{X}\right)} E
$$

Received March 8, 2004.

2000 Mathematics Subject Classification: 14F30, 14F40. 
which satisfies the following relations:

$$
\nabla(\xi x)=d \xi \otimes x+\xi \nabla x
$$

where $\xi$ and $x$ are sections of $W_{t}\left(\mathcal{O}_{X}\right)$ and $E$ respectively. The operator $\nabla$ induces maps $W_{t} \Omega_{X / S}^{l} \otimes_{W_{t}\left(\mathcal{O}_{X}\right)} E \rightarrow W_{t} \Omega_{X / S}^{l+1} \otimes_{W_{t}\left(\mathcal{O}_{X}\right)} E$, which we denote by the same letter $\nabla$. We call the de Rham-Witt connection (0.1) integrable if $\nabla^{2}=0$. A de Rham-Witt connection induces a connection on the $\mathcal{O}_{X}$-module $E \otimes_{W_{t}\left(\mathcal{O}_{X}\right)} \mathcal{O}_{X}$. If this connection is nilpotent $[\mathrm{K}]$, we call $\nabla$ nilpotent.

We denote by $W_{t}(S)$ the topological space $S$ endowed with the structure sheaf $W_{t}\left(\mathcal{O}_{S}\right)$. This is a scheme. We consider the crystalline situs $\operatorname{Crys}\left(X / W_{t}(S)\right)$ with respect to the canonical divided powers on $W_{t}(S)$.

TheOREm. Let $X / S$ be a smooth scheme. Then the category of locally free crystals of finite rank is equivalent to the category of locally free $W_{t}\left(\mathcal{O}_{X}\right)$-modules of finite rank endowed with a nilpotent, integrable de Rham-Witt connection.

This was proved by Bloch if $S$ is the spectrum of a perfect field. Let $\mathbb{E}$ be a crystal on $X$ as indicated. The starting point of the proof is the construction of Etesse [E] of the de Rham-Witt complex with coefficients in $\mathbb{E}$ over a perfect base $S$ and more recently over arbitrary $S$ in [L-Z].

Let $E$ be the value of the crystal $\mathbb{E}$ at the pd-thickening $X \rightarrow W_{t}(X)$. The crystal defines an integrable nilpotent de Rham-Witt connection (0.1) on $E$. Hence we obtain a complex:

$$
E \stackrel{\nabla}{\longrightarrow} W_{t} \Omega_{X / S}^{1} \otimes_{W_{t}\left(\mathcal{O}_{X}\right)} E \stackrel{\nabla}{\longrightarrow} W_{t} \Omega_{X / S}^{2} \otimes_{W_{t}\left(\mathcal{O}_{X}\right)} E \stackrel{\nabla}{\longrightarrow} \cdots .
$$

This is called the de Rham-Witt complex with coefficients in $E$.

We obtain a functor

$$
\mathbb{E} \longmapsto(E, \nabla)
$$

from the category of finite locally free crystals to the category of finite locally free modules with a de Rham-Witt connection.

It follows that this functor is fully faithful. Indeed, let $\mathbb{E}^{\prime}$ be a second crystal. We want to assign to a homomorphism $(E, \nabla) \rightarrow\left(E^{\prime}, \nabla^{\prime}\right)$ a homomorphism of crystals. This is a local question on $X$. Therefore we may assume that there is a Witt-lift $Y / W_{t}(S)$, i.e. $Y$ is a smooth scheme 
over $W_{t}(S)$ which lifts $X$, with a morphism $W_{t}(X) \rightarrow Y$ satisfying some properties ([L-Z, Definition 3.3]). By the Poincaré lemma [B-O] (compare $[\mathrm{L}-\mathrm{Z}, 3.56])$ we have an resolution of $\mathbb{E}$ by the complex of crystals $\mathbf{L}_{Y}\left(\Omega_{Y / W_{t}(S)} \otimes \mathbb{E}_{Y}\right)$. The comparison with the de Rham-Witt complex yields a quasi-isomorphism:

$$
\mathbb{E} \cong \mathbf{L}_{Y}\left(W_{t} \Omega_{X / S} \otimes_{W_{t}\left(\mathcal{O}_{X}\right)} E\right) .
$$

A homomorphism $(E, \nabla) \rightarrow\left(E^{\prime}, \nabla^{\prime}\right)$ induces a homomorphism of the de Rham-Witt complexes with coefficients in $E$ resp. $E^{\prime}$. Applying the functor $\mathbf{L}_{Y}$ one obtains a morphism crystals $\mathbb{E} \rightarrow \mathbb{E}^{\prime}$ from (0.3). Hence the functor $(0.2)$ is fully faithful. The assertion of the theorem is therefore local for the Zariski topology on $X$. For the proof we assume that a Witt-lift $Y$ of $X$ is given. Let $(E, \nabla)$ be an integrable de Rham-Witt connection. The arguments of Bloch with modifications in the relative case yield a locally free $\mathcal{O}_{Y}$-module $\left(M, \nabla_{M}\right)$ with an integrable connection that induces $(E, \nabla)$ by the Proposition below. If $\nabla$ is nilpotent then $\nabla_{M}$ is nilpotent and therefore defines the desired crystal $\mathbb{E}$. This proves the theorem.

We formulate the proposition in terms of rings rather than schemes. Let $R$ be a ring. Let $A$ be an $R$-algebra which is étale over a polynomial ring over $R$. We choose a Witt lift $B / W_{t}(R)$ as in the proof of [L-Z, Proposition 3.2]. The map $B \rightarrow W_{t}(A)$ induces a map of complexes:

$$
\Omega_{B / W_{t}(R)}^{\bullet} \longrightarrow W_{t} \Omega_{A / R}^{\bullet}
$$

This map is injective. Indeed, for a polynomial algebra $B$ this follows from loc.cit. Proposition 2.17. The general case is deduced by étale base change for the de Rham-Witt complex.

Proposition 1. Let $M$ be a free $W_{t}(A)$-module with an integrable connection:

$$
\nabla: M \longrightarrow W_{t} \Omega_{A / R}^{1} \otimes_{W_{t}(A)} M
$$

Then there is a basis $m_{1}, \ldots, m_{n}$ of $M$ such that

$$
\nabla\left(\begin{array}{c}
m_{1} \\
\vdots \\
m_{n}
\end{array}\right)=\Theta \otimes\left(\begin{array}{c}
m_{1} \\
\vdots \\
m_{n}
\end{array}\right),
$$

where the entries $\theta_{i j}$ of the $n \times n$-matrix $\Theta$ are in the image of the map (0.4). 
Let us assume that $S$ is noetherian and that the Frobenius morphism Frob $: S / p S \rightarrow S / p S$ is finite. This implies that the schemes $W_{t}(X), W_{t}(Y)$, $W_{t}(S)$ are noetherian.

Let $X \rightarrow Y \rightarrow S$ be smooth morphisms such that $Y / S$ is of relative dimension 1. Then we construct an exact triangle

$$
W_{t} \Omega_{Y / S}^{1} \stackrel{\mathbb{L}}{\otimes}_{W_{t}\left(\mathcal{O}_{Y}\right)} W_{t} \Omega_{X / Y}^{\bullet-1} \longrightarrow W_{t} \Omega_{X / S}^{\bullet} \longrightarrow W_{t} \Omega_{X / Y}^{\bullet}
$$

in the derived category of Zariski sheaves on $X$. The reader may wonder where the third side of this triangle is. The more precise statement is as follows: The multiplication in $W_{t} \Omega_{X / S}^{\bullet}$ induces a natural map

$$
W_{t} \Omega_{Y / S}^{1} \otimes_{W_{t}\left(\mathcal{O}_{Y}\right)} W_{t} \Omega_{X / Y}^{\bullet-1} \longrightarrow W_{t} \Omega_{X / S}^{\bullet}
$$

whose image is clearly in the kernel of the second map of $(0.7)$. We choose a complex $C$ which represents the first complex in (0.7). From the last map above we obtain a map $C \rightarrow W_{t} \Omega_{X / S}^{\bullet}$ whose image is a fortiori in the kernel of the second map of $(0.7)$. Hence we obtain a map to the mapping cone of the last morphism in (0.7):

$$
C[1] \longrightarrow W_{t} \Omega_{X / S}^{\bullet}[1] \oplus W_{t} \Omega_{X / Y}^{\bullet}
$$

The exact meaning of the words "exact triangle" is that the last morphism is a quasi-isomorphism. We note that this statement is local for the Zariski topology on $X$. Therefore the global statement (0.7) follows from the local statement Proposition 2 which we prove below.

Applying the projection formula ([H, page 106, 5.6]) to the morphism $\alpha: W_{t}(X) \rightarrow W_{t}(Y)$ we obtain an isomorphism

$$
\operatorname{R} \alpha_{*}\left(W_{t} \Omega_{Y / S}^{1} \stackrel{\mathbb{L}}{\otimes}_{W_{t}\left(\mathcal{O}_{Y}\right)} W_{t} \Omega_{X / Y}^{\bullet-1}\right)=W_{t} \Omega_{Y / S}^{1} \stackrel{\mathbb{L}}{\otimes}_{W\left(\mathcal{O}_{Y}\right)} \operatorname{R} \alpha_{*} W_{t} \Omega_{X / Y}^{\bullet-1}
$$

Hence the triangle (0.7) induces a morphism

$$
\mathrm{R} \alpha_{*} W_{t} \Omega_{X / Y}^{\bullet} \longrightarrow W_{t} \Omega_{Y / S}^{1} \stackrel{\mathbb{L}}{\otimes}_{W\left(\mathcal{O}_{Y}\right)} \operatorname{R} \alpha_{*} W_{t} \Omega_{X / Y}^{\bullet}
$$

By the comparison theorem [L-Z, Theorem 3.5] the hypercohomology groups of $\mathrm{R} \alpha_{*} W_{t} \Omega_{X / Y}$ are the crystalline cohomology groups $\mathrm{H}_{\text {cris }}^{i}\left(X / W_{t}(Y)\right)$. Let us make the assumption that these groups are locally free $W_{t}\left(\mathcal{O}_{Y}\right)$-modules for all $i$. Then we deduce the Gauß-Manin connection:

$$
\nabla: \mathrm{H}_{\mathrm{cris}}^{i}\left(X / W_{t}(Y)\right) \longrightarrow W_{t} \Omega_{Y / S}^{1} \otimes_{W\left(\mathcal{O}_{Y}\right)} \mathrm{H}_{\mathrm{cris}}^{i}\left(X / W_{t}(Y)\right) .
$$


The nilpotence of this connection follows from $[\mathrm{K}]$ since it is by definition a question for $t=1$. Since we are in the case of relative dimension 1 it is automatically integrable.

\section{§1. Witt-differentials modulo $I_{R}$}

Let $A$ be an $R$-algebra. Let $t$ be a natural number. We set $I_{R}=$ $V_{W_{t-1}}(R) \subset W_{t}(R)$. To prove the proposition we filter the Witt-differentials $W_{t} \Omega_{A / R}$ by powers of the ideal $I_{R}$. We assume that $p$ is nilpotent in $R$. Then the ideal $I_{R}$ is nilpotent.

We define

$$
\mathcal{W}_{t} \Omega_{A / R}^{\bullet}=W_{t} \Omega_{A / R}^{\bullet} / I_{R} W_{t} \Omega_{A / R}^{\bullet}
$$

This is a differential graded algebra. In particular we have:

$$
\mathcal{W}_{t} \Omega_{A / R}^{0}=\mathcal{W}_{t}(A)=W_{t}(A) / I_{R} W_{t}(A)
$$

We remark that the Teichmüller representative $x \mapsto[x]$ induces a ring homomorphism $R \rightarrow \mathcal{W}_{t}(A)$. Hence $\mathcal{W}_{t} \Omega_{A / R}^{\bullet}$ is a complex of $\mathcal{W}_{t}(A)$-modules with an $R$-linear differential

$$
d: \mathcal{W}_{t} \Omega_{A / R}^{l} \longrightarrow \mathcal{W}_{t} \Omega_{A / R}^{l+1}
$$

We denote the image of

$$
{ }^{V} W \Omega_{A / R}^{\bullet} \longrightarrow \mathcal{W}_{t} \Omega_{A / R}^{\bullet}
$$

by $\mathcal{I} \mathcal{W}_{t} \Omega_{A / R}^{\bullet}$ and in degree 0 simply $\mathcal{I}_{t}=\mathcal{I}_{\mathcal{W}_{t}} \Omega_{A / R}^{0}={ }^{V} W_{t-1}(A) / I_{R} W_{t}(A)$ (note that there is no Verschiebung on $\mathcal{W}_{\bullet} \Omega_{A / R}$, and we have an action of $F$ only if $p R=0)$. $\mathcal{I} \mathcal{W}_{t} \Omega_{A / R}^{\bullet}$ is an ideal in the differential graded algebra $\mathcal{W}_{t} \Omega_{A / R}^{\bullet}$. We obviously have

$$
\left(\mathcal{I} \mathcal{W}_{t} \Omega_{A / R}^{\bullet}\right)^{2}=0
$$

because $V_{\omega} \cdot V_{\eta}=p \cdot V_{(\omega \eta)}={ }^{V} 1 \cdot{ }^{V}(\omega \eta)$.

We define a second ideal $\mathcal{F} \mathcal{W}_{t} \Omega_{A / R}^{\bullet}$ as the kernel of the homomorphism of differential graded algebras

$$
0 \longrightarrow \mathcal{F} \mathcal{W}_{t} \Omega_{A / R}^{\bullet} \longrightarrow \mathcal{W}_{t} \Omega_{A / R}^{\bullet} \longrightarrow \Omega_{A / R}^{\bullet} \longrightarrow 0
$$

We have

$$
\mathcal{I W}_{t} \Omega_{A / R}^{\bullet}+d \mathcal{I W}_{t} \Omega_{A / R}^{\bullet} \subseteq \mathcal{F} \mathcal{W}_{t} \Omega_{A / R}^{\bullet}
$$


If $A / R$ is smooth, we will see that (1.2) is a direct sum decomposition and that the differential $d$ is injective, i.e. we have

$$
\mathcal{I W}_{t} \Omega_{A / R}^{\bullet} \oplus d \mathcal{I}_{t} \Omega_{A / R}^{\bullet}=\mathcal{F W}_{t} \Omega_{A / R}^{\bullet}
$$

and there is an exact sequence

$$
0 \longrightarrow \mathcal{I W}_{t} \Omega_{A / R}^{\bullet} \stackrel{d}{\longrightarrow} \mathcal{W}_{t} \Omega_{A / R}^{\bullet} / \mathcal{I} \mathcal{W}_{t} \Omega_{A / R}^{\bullet} \longrightarrow \Omega_{A / R}^{\bullet} \longrightarrow 0 .
$$

A Witt-lift $B / W_{t}(R)$ with $B \rightarrow W_{t}(A)$ defines a map $\Omega_{B / W_{t}(R)}^{\bullet} \rightarrow W_{t} \Omega_{A / R}^{\bullet}$ which induces - by reduction modulo $I_{R}-$ a map $\Omega_{A / R}^{\bullet} \rightarrow \mathcal{W}_{t} \Omega_{A / R}^{\bullet}$, hence a Witt-lift defines a splitting of the sequence (1.1). In particular a Witt-lift defines (for $A / R$ smooth) a direct decomposition

$$
\begin{aligned}
& \mathcal{W}_{t} \Omega_{A / R}^{l}=\mathcal{I}_{\mathcal{W}_{t}} \Omega_{A / R}^{l} \oplus d \mathcal{I} \mathcal{W}_{t} \Omega_{A / R}^{l-1} \oplus \Omega_{A / R}^{l} \\
& \omega=\eta_{1}+d \eta_{2}+\eta_{3} .
\end{aligned}
$$

We write $\delta \omega=\eta_{2}$. Let pr: $\mathcal{W}_{t} \Omega_{A / R}^{l} \rightarrow \mathcal{W}_{t} \Omega_{A / R}^{l}$ be the projection on $\Omega_{A / R}^{l}$. Then pr is homotopic to the identity:

$$
(\mathrm{id}-\mathrm{pr})(\omega)=d \delta \omega+\delta d \omega
$$

Hence the complexes $\mathcal{W}_{t} \Omega_{A / R}^{\bullet}$ and $\Omega_{A / R}^{\bullet}$ are homotopy equivalent.

Remark. The differential $d: \mathcal{I W}_{t} \Omega_{A / R}^{\bullet} \rightarrow \mathcal{F W}_{t} \Omega_{A / R}^{\bullet} / \mathcal{I} \mathcal{W}_{t} \Omega_{A / R}^{\bullet}$ is a homomorphism of $\mathcal{W}_{t} \Omega_{A / R^{\bullet}}$ modules for the action from the right:

$$
d\left({ }^{V} \eta \omega\right)=d^{V} \eta \omega+(-1)^{\operatorname{deg} \eta V} \eta d \omega \equiv d^{V} \eta \omega \quad \bmod \mathcal{I W}_{t} \Omega_{A / R}^{\bullet}
$$

It remains to prove the following.

LEMMA 1. If $A / R$ is smooth, then we have

$$
\mathcal{I W}_{t} \Omega_{A / R}^{\bullet} \oplus d \mathcal{I} \mathcal{W}_{t} \Omega_{A / R}^{\bullet}=\mathcal{F} \mathcal{W}_{t} \Omega_{A / R}^{\bullet}
$$

Proof. By $[\mathrm{L}-\mathrm{Z},(2.43)]$ it suffices to show that the sum is a direct sum. For this it suffices to show that the $W_{t}(A)$-module homomorphism

$$
d: \mathcal{I W}_{t} \Omega_{A / R}^{\bullet} \longrightarrow \mathcal{F} \mathcal{W}_{t} \Omega_{A / R}^{\bullet} / \mathcal{I W}_{t} \Omega_{A / R}^{\bullet}
$$

is an isomorphism. 
Let $P=R\left[T_{1}, \ldots, T_{d}\right]$ be a polynomial algebra. Assume that $A$ is étale over $P$. We obtain (1.5) from the corresponding morphism for $P$ by tensoring with the étale morphism $W_{t}(P) \rightarrow W_{t}(A)$. Hence we may assume $A=R\left[T_{1}, \ldots, T_{d}\right]$. In this case each element of $W_{t} \Omega_{A / R}^{l}$ is a unique sum of basic Witt differentials $e\left(\xi, k, I_{0}, \ldots, I_{l}\right)$ by [L-Z, Proposition 2.17]. Recall that a weight $k:[1, d] \in \mathbb{Z}_{\geq 0}[1 / p]$ in such a sum has denominator $p^{u} \leq p^{t}$. We denote by $\bar{e}\left(\xi, k, I_{0}, \ldots, I_{l}\right)$ the images of the basic Witt differentials in $\mathcal{W}_{t} \Omega_{A / R}^{l}$. Then the claim in Lemma 1 follows from the following.

Lemma 2. Let $\omega \in \mathcal{W}_{t} \Omega_{P / R}^{l}$. Then $\omega$ has a unique representation

$$
\omega=\sum_{k \text { integral }} \bar{e}\left(\xi_{k}, k, I_{0}, \ldots, I_{l}\right)+\sum_{k \text { frac }} \bar{e}\left(\xi_{k}, k, I_{0}, \ldots, I_{l}\right)
$$

with $\xi_{k} \in R$ for $k$ integral and $\xi_{k} \in V^{u} W_{t-u}(R) / p^{V^{u}} W_{t-u}(R)$ for $k$ fractional such that $p^{u}$ is the denominator of $k$. The elements in $\mathcal{I W}_{t} \Omega_{A / R}^{l}$ are the elements with a representation

$$
\omega=\sum_{\substack{I_{0} \neq \varnothing \\ k \text { frac }}} \bar{e}\left(\xi_{k}, k, I_{0}, \ldots, I_{l}\right) .
$$

Proof. Lemma 2 follows from loc.cit. Proposition 2.17 in conjunction with Proposition 2.5.

\section{§2. Integrable connections on $\mathcal{W}_{t}(A)$-modules}

Let $\mathcal{M}$ be a free $\mathcal{W}_{t}(A)$-module of rank $n$. We consider a connection

$$
\nabla: \mathcal{M} \longrightarrow \mathcal{W}_{t} \Omega_{A / R}^{1} \otimes_{\mathcal{W}_{t}(A)} \mathcal{M}
$$

and call $\nabla$ integrable, if $\nabla^{2}: \mathcal{M} \rightarrow \mathcal{W}_{t} \Omega_{A / R}^{2} \otimes_{\mathcal{W}_{t}(A)} \mathcal{M}$ vanishes. Let $m_{1}, \ldots, m_{n}$ be a basis of $\mathcal{M}$. We write

$$
\nabla\left(\begin{array}{c}
m_{1} \\
\vdots \\
m_{n}
\end{array}\right)=\Theta \otimes\left(\begin{array}{c}
m_{1} \\
\vdots \\
m_{n}
\end{array}\right)
$$

where $\Theta$ is a $n \times n$-matrix with entries in $\mathcal{W}_{t} \Omega_{A / R}^{1}$. We abbreviate (2.1) as $\nabla(\underline{m})=\Theta \otimes \underline{m}$, and we call $\Theta$ the connection matrix. 
LEMmA 3. Let $(\mathcal{M}, \nabla)$ be an integrable connection. Then there is a basis $\underline{m}$ of $\mathcal{M}$, such that the connection matrix has coefficients in $\Omega_{A / R}^{1} \subset$ $\mathcal{W}_{t} \Omega_{A / R}^{1}$

Proof. We first show the analogous claim for the induced connection

$$
\bar{\nabla}: \mathcal{M} \longrightarrow\left(\mathcal{W}_{t} \Omega_{A / R}^{1} / \mathcal{I} \mathcal{W}_{t} \Omega_{A / R}^{1}\right) \otimes_{\mathcal{W}_{t}(A)} \mathcal{M}
$$

By (1.4) the connection matrix $\bar{\Theta}$ of $\bar{\nabla}$ is of the form $\bar{\Theta}=\omega+d \rho$ with $\omega \in M\left(n \times n, \Omega_{A / R}^{1}\right)$ and $\rho \in M(n \times n, \mathcal{I})$. Then define $\underline{m}^{\prime}=(1-\rho) \underline{m}$. We get

$$
\begin{aligned}
\bar{\nabla} \underline{m}^{\prime} & =d(1-\rho) \otimes \underline{m}+(1-\rho) \omega \otimes \underline{m}+(1-\rho) d \rho \otimes \underline{m} \\
& =\omega \otimes \underline{m}^{\prime}-\rho d \rho \otimes \underline{m} \equiv \omega \otimes \underline{m}^{\prime} \quad \bmod \mathcal{I} \mathcal{W}_{t} \Omega_{A / R}^{1} \otimes \mathcal{M} .
\end{aligned}
$$

Hence we can find a basis of $\mathcal{M}$ such thet $\bar{\Theta}$ has coefficients in $\Omega_{A / R}^{1}$. Then the connection matrix $\Theta$ with respect to this basis has the form $\Theta=\omega+\eta$ with $\omega \in M\left(n \times n, \Omega_{A / R}^{1}\right)$ and $\eta \in M\left(n \times n, \mathcal{I} \mathcal{W}_{t} \Omega_{A / R}^{1}\right)$. Then we have

$$
\nabla^{2} \underline{m}=d \omega \otimes \underline{m}+d \eta \otimes \underline{m}+\omega^{2} \otimes \underline{m}+\omega \eta \otimes \underline{m}+\eta \omega \otimes \underline{m}+\eta^{2} \otimes \underline{m} .
$$

We consider the matrix of this map $\nabla^{2}: \mathcal{M} \rightarrow \mathcal{W}_{t} \Omega_{A / R}^{2} \otimes \mathcal{M}$ corresponding to the decomposition (1.4)

$$
(\omega \eta+\eta \omega) \oplus(d \eta) \oplus\left(d \omega+\omega^{2}\right) .
$$

We remark that $\eta^{2}=0$ because $\left(\mathcal{I}_{\mathcal{W}_{t}} \Omega_{A / R}\right)^{2}=0$. Since $\nabla$ is integrable, all summands in (2.3) are zero, in particular $d \eta=0$, hence $\eta=0$. So the connection matrix $\Theta$ has coefficients in $\Omega_{A / R}^{1}$. Lemma 3 follows.

\section{§3. Proof of Proposition 1}

For $A / R$ smooth we consider the filtration $I_{R}^{m} W_{t} \Omega_{A / R}^{\bullet}$ of the de RhamWitt complex. We set

$$
\mathcal{W}_{t}^{(l)} \Omega_{A / R}^{\bullet}=W_{t} \Omega_{A / R}^{\bullet} / I_{R}^{l} W_{t} \Omega_{A / R}^{\bullet}
$$

and

$$
\mathcal{G}_{l}^{\bullet}=I_{R}^{l} W_{t} \Omega_{A / R}^{\bullet} / I_{R}^{l+1} W_{t} \Omega_{A / R}^{\bullet}
$$

Then we have a canonical map

$$
p^{l}: \mathcal{I} \mathcal{W}_{t} \Omega_{A / R}^{\bullet} \longrightarrow \mathcal{G}_{l}^{\bullet} \text {. }
$$


Assume that $B \rightarrow W_{t}(A)$ is a Witt-lift over $W_{t}(R)$ and $\Omega_{B / W_{t}(R)}^{\bullet} \rightarrow$ $W_{t} \Omega_{A / R}^{\bullet}$ the induced map. Similarly to (1.4) we find a direct sum decomposition

$$
\mathcal{G}_{l}^{\bullet}=p^{l} \mathcal{I}_{\mathcal{W}_{t}} \Omega_{A / R}^{\bullet} \oplus p^{l} d \mathcal{I} \mathcal{W}_{t} \Omega_{A / R}^{\bullet-1} \oplus I_{R}^{l} / I_{R}^{l+1} \otimes_{R} \Omega_{A / R}^{\bullet}
$$

We start with the connection $\nabla: M \rightarrow W_{t} \Omega_{A / R}^{1} \otimes_{W_{t}(A)} M$ of Proposition 1. This induces connections on $\mathcal{M}_{l}=M / I_{R}^{l} M$

$$
\nabla^{(l)}: \mathcal{M}_{l} \longrightarrow \mathcal{W}_{t}^{(l)} \Omega_{A / R} \otimes_{\mathcal{W}_{t}^{(l)}(A)} \mathcal{M}_{l}
$$

Assume we have a basis $\underline{\tilde{m}}$ of $\mathcal{M}_{l}$ such that

$$
\nabla^{(l)} \underline{\tilde{m}}=\Theta \otimes \underline{\widetilde{m}} \quad \text { with } \quad \Theta \in M\left(n \times n, \Omega_{B / W_{t}(R)}^{1} / I_{R}^{l} \Omega_{B / W_{t}(R)}^{1}\right) .
$$

Since the existence of such a base was shown for $l=1$ in Section 2 it is enough to show the following:

Claim. There is a lifting $\underline{m}$ of $\underline{\tilde{m}}$ to $\mathcal{M}_{l+1}$ such that (3.3) holds for $l+1$.

Proof. Let $\underline{m}$ be any lifting. Then (3.2) implies

$$
\nabla^{(l+1)} \underline{m}=\Theta \otimes \underline{m}+p^{l} \eta \otimes \underline{m}+p^{l} d \rho \otimes \underline{m}
$$

with $\rho, \eta$ having coefficients in $\mathcal{I} \mathcal{W}_{t} \Omega^{0 \text { or } 1}$ and $\Theta$ in $\Omega_{B / W_{t}(R)}^{1} / I_{R}^{l+1} \Omega_{B / W_{t}(R)}^{1}$. Now we consider the induced connection

$$
\begin{gathered}
\widetilde{\nabla}^{(l+1)}: \mathcal{M}_{l+1} \longrightarrow \mathcal{W}_{t}^{(l+1)} \Omega_{A / R} / p^{l} \mathcal{I} \mathcal{W}_{t} \Omega_{A / R}^{\bullet} \otimes_{\mathcal{W}_{t}^{(l+1)}(A)} \mathcal{M}_{l+1} . \\
\widetilde{\nabla}^{(l+1)}(\underline{m})=\Theta \otimes \underline{m}+p^{l} d \rho \otimes \underline{m} .
\end{gathered}
$$

Choose $\underline{m}^{\prime}=\left(1-p^{l} \rho\right) \underline{m}$ as new basis. As in (2.2) we can write

$$
\nabla^{(l+1)} \underline{m}^{\prime}=\Theta \otimes \underline{m}^{\prime}+p^{l} \eta \otimes \underline{m}^{\prime} .
$$

We compute

$$
\left(\nabla^{(l+1)}\right)^{2} \underline{m}^{\prime}=\left(d \Theta+\Theta^{2}\right) \otimes \underline{m}^{\prime}+p^{l}(\Theta \eta+\eta \Theta) \otimes \underline{m}^{\prime}+p^{l} d \eta \otimes \underline{m}^{\prime} .
$$

Since $\nabla^{(l)}$ is integrable, we have $d \Theta+\Theta^{2} \in M\left(n \times n, I_{R}^{l} / I_{R}^{l+1} \otimes_{R} \Omega_{A / R}^{2}\right)$. Since $p^{l}(\Theta \eta+\eta \Theta)$ has coefficients in $p^{l} \mathcal{I} \mathcal{W}_{t} \Omega_{A / R}^{2}$, the decomposition (3.2) implies that $p^{l} d \eta=0$ and hence $p^{l} \eta=0$. The claim follows. 


\section{$\S 4$. The Gauß-Manin connection in the smooth case}

Consider ring homomorphisms $R \rightarrow S \rightarrow T$. In the graded pro-algebra $W_{t} \Omega_{T / R}^{\bullet}$ we can consider the ideal $\mathcal{I}_{t}$ generated by all $d s$ for $s \in W_{t}(S)$. Then $\mathcal{I}_{t}$ is invariant under $d$, and the pro-ideal $\left\{\mathcal{I}_{t}\right\}$ is invariant under $F$ and $V$. We show the invariance under $F$. A general element of $\mathcal{I}_{t}$ is a sum of elements of the form $\omega d s$ with $\omega \in W_{t} \Omega_{T / R}$ and $s \in W_{t}(S)$. Let $s=\left(x_{0}, x_{1}, x_{2}, \ldots\right)$ and $\rho=\left(x_{1}, x_{2}, x_{3}, \ldots\right)$. Then ${ }^{F} d s=\left[x_{0}^{p-1}\right] d\left[x_{0}\right]+d \rho$ and we have

$$
F_{(\omega d s)}={ }^{F} \omega^{F} d s={ }^{F} \omega\left[x_{0}^{p-1}\right] d\left[x_{0}\right]+{ }^{F} \omega d \rho .
$$

This is an element in $\mathcal{I}_{t-1}$.

The canonical epimorphism $W_{t} \Omega_{T / R}^{\bullet} \rightarrow W_{t} \Omega_{T / S}^{\bullet}$ factors obviously through a map

$$
W_{t} \Omega_{T / R}^{\bullet} / \mathcal{I}_{t} \longrightarrow W_{t} \Omega_{T / S}^{\bullet}
$$

The properties of $\mathcal{I}_{t}$ show that the left hand side is a $F-V$-pro-complex in which $d$ becomes a $W(S)$-linear map. Hence the map (4.1) has a section by the universal property of $W_{t} \Omega_{T / S}^{\bullet}$. This section is surjective, because it maps $d t$, for $t \in W_{t}(T)$, to the same element in $W_{t} \Omega_{T / R}^{\bullet}$, and these differentials generate $W_{t} \Omega_{T / R}$. Therefore the map (4.1) is an isomorphism and we have an exact sequence

$$
0 \longrightarrow \mathcal{I}_{t} \longrightarrow W_{t} \Omega_{T / R}^{\bullet} \longrightarrow W_{t} \Omega_{T / S}^{\bullet} \longrightarrow 0 .
$$

We also have a sequence of maps, in fact a complex for each $k \geq 1$

$$
W_{t} \Omega_{S / R}^{1} \otimes_{W_{t}(S)} W_{t} \Omega_{T / R}^{k-1} \longrightarrow W_{t} \Omega_{T / R}^{k} \longrightarrow W_{t} \Omega_{T / S}^{k} \longrightarrow 0 .
$$

Let now $S / R$ be smooth of relative dimension 1 , hence $W \Omega_{S / R}^{i}=0$ for $i \geq 2$. This implies that the maps $W_{t} \Omega_{S / R}^{1} \otimes_{W_{t}(S)} \mathcal{I}_{t} \rightarrow W_{t} \Omega_{T / R}^{\bullet}$ are zero (because $d s_{1} d s_{2}=0$ for $s_{1}, s_{2} \in W_{t}(S)$ ). The sequence (4.2) factors through a sequence of complexes

$$
W_{t} \Omega_{S / R}^{1} \otimes_{W(S)} W_{t} \Omega_{T / S}^{\bullet}[-1] \longrightarrow W_{t} \Omega_{T / R}^{\bullet} \longrightarrow W_{t} \Omega_{T / S}^{\bullet}
$$

From now on we assume that $p$ is nilpotent in $R$, and that the Frobenius morphism on $R / p R$ is a finite map. Moreover all morphisms $R \rightarrow S \rightarrow T$ will be smooth and $S / R$ will be of relative dimension 1 . In the following we will prove that (4.3) becomes a distinguished triangle when we replace the tensor product on the left hand side by the derived tensor product. More precisely we show: 
Proposition 2. Under the above assumptions the sequence (4.3) defines a distinguished triangle in the derived category of $W_{n}(R)$-modules.

$$
W_{t} \Omega_{S / R}^{1} \stackrel{\mathbb{L}}{\otimes}{ }_{W(S)} W_{t} \Omega_{T / S}^{\bullet}[-1] \longrightarrow W_{t} \Omega_{T / R}^{\bullet} \longrightarrow W_{t} \Omega_{T / S}^{\bullet}
$$

The question is local for the étale topology on $\operatorname{Spec} T$. To see this we choose a number $m$ such that $p^{m}$ annihilates $W_{t}(R)$ and consequently all modules in the triangle above. The individual modules in the complexes involved are $W_{t}(T)$-modules. We consider them as $W_{t+m}(T)$-modules via restriction of scalars $F^{m}: W_{t+m}(T) \rightarrow W_{t}(T)$. Then the complexes involved are complexes of $W_{t+m}(T)$-modules with linear differentials. Let $T \rightarrow T^{\prime}$ be a faithfully flat étale morphism. Then the same holds for $W_{t+m}(T) \rightarrow$ $W_{t+m}\left(T^{\prime}\right)$. The étale base change for the de Rham-Witt complex shows that

$$
W_{t+m}\left(T^{\prime}\right) \otimes_{W_{t+m}(T)} W_{t} \Omega_{T / R}^{\bullet} \cong W_{t} \Omega_{T^{\prime} / R}
$$

as complexes in the category of $W_{t+m}\left(T^{\prime}\right)$-modules. Together with the corresponding fact for $T / R$ replaced by $T / S$ this shows that the assertion is local with respect to the étale topology on $\operatorname{Spec} T$. Hence we may assume that the map $S \rightarrow T$ of $R$-algebras fits into a square

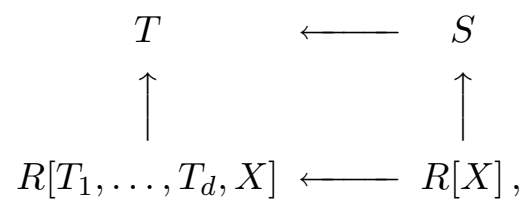

where the vertical maps are étale. Let $S^{\prime}=R[X]$. We first reduce to the case that $S=S^{\prime}$. Suppose that (4.4) holds for the diagram of rings $R \rightarrow S^{\prime} \rightarrow T$. Since any $W_{t}\left(S^{\prime}\right)$-linear derivation on $W_{t}(T)$ vanishes on $W_{t}(S)$ (because $W_{t}(S)$ is étale over $W_{t}\left(S^{\prime}\right)$ ) it follows from the universal property of $\left\{W_{t} \Omega_{T / S}^{\bullet}\right\}$ as $F-V$-pro-complex, that $W_{t} \Omega_{T / S}^{\bullet}=W_{t} \Omega_{T / S^{\prime}}^{\bullet}$. The left hand side in (4.4) may be rewritten as follows:

$$
\begin{aligned}
W_{t} \Omega_{S / R}^{1} \stackrel{\mathbb{L}}{\otimes}_{W(S)} W_{t} \Omega_{T / S}^{\bullet}=\left(W_{t} \Omega_{S^{\prime} / R}^{1} \otimes_{W_{t}\left(S^{\prime}\right)} W_{t}(S)\right) \stackrel{\mathbb{L}}{\otimes_{W(S)}} W_{t} \Omega_{T / S}^{\bullet} \\
=W_{t} \Omega_{S^{\prime} / R}^{1} \stackrel{\mathbb{L}}{\otimes}_{W\left(S^{\prime}\right)} W_{t} \Omega_{T / S}^{\bullet}=W_{t} \Omega_{S^{\prime} / R}^{1} \stackrel{\mathbb{L}}{\otimes}_{W\left(S^{\prime}\right)} W_{t} \Omega_{T / S^{\prime}}^{\bullet}
\end{aligned}
$$

Hence we may assume that $S=R[X]$. By the étale base change property of the de Rham-Witt complex we may also assume $T=S\left[Y_{1}, \ldots, Y_{d}\right]$ is a 
polynomial algebra as well. We will show that the derived tensor product in the triangle (4.4) is quasi-isomorphic to the kernel of the second map.

Let $\widetilde{T}=W_{t}(S)\left[Y_{1}, \ldots, Y_{d}\right]$ be the canonical Witt-lift of $T$ with the map $\widetilde{T} \stackrel{\sigma}{\rightarrow} W_{t}(T), Y_{i} \mapsto\left[Y_{i}\right]$. By [L-Z, (3.8)] this induces a quasi-isomorphism $\Omega_{\widetilde{T} / W_{t}(S)}^{\bullet} \rightarrow W_{t} \Omega_{T / S}^{\bullet}$. Hence we obtain a morphism:

$$
W_{t} \Omega_{S / R}^{1} \otimes_{W_{t}(S)} \Omega_{\widetilde{T} / W_{t}(S)}^{\bullet}[-1] \longrightarrow W_{t} \Omega_{S / R}^{1} \otimes_{W_{t}(S)} W_{t} \Omega_{T / S}^{\bullet}[-1] .
$$

This arrow represents the derived tensor product $W_{t} \Omega_{S / R}^{1} \stackrel{\mathbb{L}}{\otimes_{W(S)}} W_{t} \Omega_{T / S}^{\bullet}[-1]$. From (4.6) we obtain a morphism

$$
W_{t} \Omega_{S / R}^{1} \otimes_{W_{t}(S)} \Omega_{\widetilde{T} / W_{t}(S)}^{\bullet}[-1] \longrightarrow W_{t} \Omega_{T / R}^{\bullet},
$$

which factors through $\mathcal{I}_{t}$. Therefore we have to show that (4.7) induces a quasi-isomorphism.

$$
W_{t} \Omega_{S / R}^{1} \otimes_{W_{t}(S)} \Omega_{\widetilde{T} / W_{t}(S)}^{\bullet}[-1] \longrightarrow \mathcal{I}_{t} .
$$

We consider Witt differentials in $\mathcal{I}_{t}$ of the following type: To denote basic Witt differentials in $W_{t} \Omega_{T / R}^{\bullet}$ we use weight functions $k:[0, d] \rightarrow$ $\mathbb{Z}_{\geq 0}\left[1 / p^{t-1}\right]$, where the argument 0 corresponds to the variable $X$. We will consider basic Witt differentials $e(\xi, k, \mathcal{P})=e_{t}(\xi, k, \mathcal{P})$ as in [L-Z, Proposition 2.17].

Definition 1. Let $T$ be a polynomial algebra over $R$. We say a differential $\omega \in W_{t} \Omega_{T / R}^{\bullet}$ is of weight $k$ if it is a sum of basic Witt differentials of the type $e(\xi, k, \mathcal{P})$.

The $W_{t}(R)$-module $W_{t} \Omega_{T / R}^{\bullet}$ is the direct sum of its graded pieces $W_{t} \Omega(k)_{T / R}^{\bullet}$. The operators $F, V, d$ respect this graduation as follows ([L-Z, Proposition 2.5]):

$$
\begin{aligned}
& F: W_{t} \Omega(k)_{T / R}^{\bullet} \longrightarrow W_{t-1} \Omega(p k)_{T / R}^{\bullet} \\
& V: W_{t} \Omega(k)_{T / R}^{\bullet} \longrightarrow W_{t+1} \Omega(k / p)_{T / R}^{\bullet} \\
& d: W_{t} \Omega(k)_{T / R}^{\bullet} \longrightarrow W_{t} \Omega(k)_{T / R}^{\bullet}
\end{aligned}
$$

A differential $\omega$ in the integral part may be indentified with a differential of the de Rham complex $\Omega_{W(R)[X, \underline{T}] / W(R)}$. In this case the concept of weight above coincides with the usual concept for the de Rham complex of a polynomial algebra (compare [L-Z, Sections 2.1 and 3.3]). 
Lemma 4. Let $\omega_{1}, \omega_{2} \in W_{t} \Omega_{T / R}^{\bullet}$ be differentials of weight $k_{1}$ respectively $k_{2}$. Then the product $\omega_{1} \omega_{2}$ has weight $k_{1}+k_{2}$.

Proof. By the remark above this is clear if both weights $k_{1}$ and $k_{2}$ are integral. In the general case we assume that $\omega_{i}$ for $i=1,2$ are basic Witt differentials. We write $\omega_{i}={ }^{V_{i}} \omega_{i}^{\prime}$ or $d^{V^{u_{i}}} \omega_{i}^{\prime}$ for $i=1,2$ and $\omega_{1}^{\prime}$ and $\omega_{2}^{\prime}$.

Let us begin with the case $\omega_{1}=V^{u_{1}} \omega_{1}^{\prime}$ and $\omega_{2}=V^{u_{2}} \omega_{2}^{\prime}$. Without loss of generality we may assume that $u_{1} \geq u_{2}$. Our assertion follows from the formula:

$$
\omega_{1} \omega_{2}=V^{u_{1}}\left(\omega_{1}^{\prime} p^{\left.u_{2} F^{u_{1}-u_{2}} \omega_{2}^{\prime}\right) .}\right.
$$

Next we show that the expression ${ }^{u_{1}} \omega_{1}^{\prime} d^{V^{u_{2}} \omega_{2}^{\prime}}$ is of weight $k_{1}+k_{2}$. Using the Leibniz rule and what we already proved, we may assume that $u_{1} \geq u_{2}$. Then the expression becomes:

$$
V^{u_{1}}\left(\omega_{1}^{\prime} F^{u_{1}-u_{2}} d \omega_{2}^{\prime}\right) .
$$

The assertion follows from the integral case. The rest of the proof is similiar.

From now on we assume $k(0)=0$, i.e. $X$ doesn't appear in the basic Witt differentials. We will denote by $l \in \mathbb{Z}\left[1 / p^{t-1}\right]$ a number with denominator $p^{u(l)}$, such that $u(l) \geq 0$.

Then we consider Witt differentials of the following 3 types:

a) $e(\xi, k, \mathcal{P})^{F^{r}} d\left[X^{s}\right]$, where $r, s \in \mathbb{Z}_{\geq 0}, s$ not divisible by $p, \xi \in W_{t}(R)$.

b) $e(\xi, k, \mathcal{P}) d^{V^{u(l)}}\left[X^{p^{u(l)} l}\right]$, where $u(k) \geq u(l)>0$.

c) $e(1, k, \mathcal{P}) d^{V^{u(l)}}\left(\eta\left[X^{p^{u(l)}}\right]\right)$, where $u(k)<u(l), \eta \in W_{t-u(l)}(R)$.

Lemma 5. Each element in $\mathcal{I}_{t}$ is a unique sum of elements of the type above.

Proof. We begin showing that an element of $\mathcal{I}_{t}$ may be expressed as a sum of elements of type a), b), c). $\mathcal{I}_{t}$ is generated as an abelian group by elements of the form $\omega d s$ where $\omega \in W_{t} \Omega_{T / R}^{\bullet}$ is a basic Witt differential and $s \in W_{t}(S)$. If $\omega$ doesn't contain the variable $X$ we are almost done, because an element $d s$ may be written by the one-dimensional case of [L-Z] loc.cit. in the form

$$
\xi^{F^{r}} d\left[X^{s}\right], \quad \text { or } \quad d^{V^{u(l)}}\left(\eta\left[X^{p^{u(l)}}\right]\right) .
$$


Assume for example that $d s$ is of the second type in (4.9) and $u(k) \geq$ $u(l)$. We may write $\omega=V^{u(k)} \omega_{1}$. We write:

$$
\omega d s=V^{u(l)}\left(V^{u(k)-u(l)} \omega_{1} d \eta\left[X^{p^{u(l)}}\right]\right) .
$$

This permits to place the constant $\eta$ in the Witt differential $\omega$. Hence we obtain the type b) above. The type c) is obtained in a similiar way.

Next we consider the case, where $\omega$ contains the variable $X$. Let us assume that $\omega$ is integral, i.e. is in the de Rham complex of the lifting $W_{t}(R)[X, \underline{T}]$. In this case $\omega=[X]^{e} \omega^{\prime}$ or $\omega=F^{t} d\left([X]^{e}[\underline{Y}]^{f}\right) \omega^{\prime}$. If we have the first equality we just have to write $[X]^{e} d s$ as a sum of basic Witt differentials. If the second equality holds we find by the Leibniz rule:

$$
F^{t} d\left([X]^{e}[\underline{Y}] \underline{f}\right) d s=F^{t} d([\underline{Y}] \underline{f}) F^{t}[X]^{e} d s .
$$

This expression may be written as a sum of Witt differentials of type a). Now assume that $\omega$ is not integral and write $\omega=V^{u} \omega^{\prime}$ of $d^{V^{u}} \omega^{\prime}$ for some integral $\omega^{\prime}$. If the first equation holds we write:

$$
\omega d s=V^{u}\left(\omega^{\prime} F^{u} d s\right) .
$$

We apply what we proved to the expression in brackets. Then we remark that $V^{u}$ applied to an element of type a), b), c). is again an element of this type by standard formulas [L-Z, Definition 1.4 and (1.16)]. By the former case this expression may be written in the form $\omega_{1} d s_{1}$, where $\omega_{1}$ doesn't contain the variable $X$. The case $\omega=d^{V^{u} \omega^{\prime}}$ is similiar.

To show the uniqueness we remark that the elements in the list a), b), c) above are all homogenous for the weight graduation by Lemma 4 . Therefore we have to show that for a fixed weight a sum of these elements over different weights can't be zero, if not all members of this sum are zero.

Consider the case a) and assume that $k$ is integral. Note that $r, s$ are fixed in the sum. Since we are in the de Rham complex of a polynomial algebra the multiplication by ${ }^{F^{r}} d\left[X^{s}\right]$ can't kill a differential form, which doesn't depend on $X$. By the independence of the $e(\xi, k, \mathcal{P})$ we conclude that all members of the sum must be zero. If $k$ is not integral we have a sum of basic Witt differentials of type $V^{u} e\left(\xi, k^{\prime}, \mathcal{P}\right)$ and $d^{V^{u}} e\left(\xi, k^{\prime}, \mathcal{P}\right)$ with $k^{\prime}$ primitive integral multiplied with $F^{r} d\left[X^{s}\right]$. Assume that this expression is zero and apply $d$. Then we obtain for the remaining sum:

$$
d^{V^{u(k)}}\left(\sum_{\mathcal{P}} e\left(\xi_{\mathcal{P}}, k^{\prime}, \mathcal{P}\right)^{F^{(r+u)}} d\left[X^{s}\right]\right)=0 .
$$


We claim that the sum is zero. Indeed by Lemma 4 this sum is a sum of basic Witt elements of primitive integral weight. There the operator $d^{V^{u(k)}}$ acts injectively (compare: [L-Z, Theorem 2.24]). Hence by the integral case all terms in this sum are zero. Going back to the situation before applying $d$, we see that no terms $V^{u(k)} e\left(\xi, k^{\prime}, \mathcal{P}\right)$ can appear in our sum. Doing our argument again the case a) follows.

The same argument applies if we want to show our assertion in case b).

Finally we consider the case of a sum of elements of type c). We use the Leibniz rule:

$$
\begin{aligned}
& e(1, k, \mathcal{P}) d^{V^{u(l)}}\left(\eta\left[X^{p^{u(l)}}\right]\right) \\
& \quad= \pm d\left(e(1, k, \mathcal{P})^{V^{u(l)}}\left(\eta\left[X^{p^{u(l)}}\right]\right)\right) \mp V^{u(l)}\left(\eta\left[X^{p^{u(l)} l}\right]\right) e(1, k, \mathcal{P})
\end{aligned}
$$

The right hand side is a sum of two basic Witt differentials for different partitions. Indeed for the first summand the first intervall of the partition is zero but for the second not. Therefore in this case the assertion follows from the independence of basic Witt elements.

We will now finish the proof of Proposition 2. It suffices to show that the map (4.8) is a quasi-isomorphism. We know by [L-Z, Proposition 2.1] that the elements $e(1, k, \mathcal{P}) \in \Omega_{\widetilde{T} / W_{t}(S)}^{\bullet}$ form a basis over $W_{t}(S)$, where $k:[1, d] \rightarrow \mathbb{Z}_{\geq 0}$ runs over all integral weights. Hence any element in the abelian group $W_{t} \Omega_{S / R}^{1} \otimes_{W_{t}(S)} \Omega_{\widetilde{T} / W_{t}(S)}^{\bullet}$ is a unique sum of elements of the following type:

1) $\xi^{F^{r}} d\left[X^{s}\right] \otimes e(1, k, \mathcal{P})$, where $r, s \in \mathbb{Z}_{\geq 0}, s$ not divisible by $p, \xi \in W_{t}(R)$.

2) $d^{V^{u(l)}}\left(\eta\left[X^{p^{u(l)}}\right]\right) \otimes e(1, k, \mathcal{P})$, where $0<u(l), \eta \in W_{t-u(l)}(R)$.

These elements are mapped by (4.8) exactly to elements of type a) and c) above with $k$ integral. Therefore an element in the cokernel $\overline{\mathcal{I}}_{t}$ of (4.8) is a unique sum of elements of type a), b), c) with $k$ not integral. By [L-Z, Proposition 2.6] the cycles in the complex $\overline{\mathcal{I}_{t}}$ are generated by those elements, where the first intervall of the partition $\mathcal{P}$ is empty. But these elements are also boundaries. This proves Proposition 2.

\section{REFERENCES}

[B] P. Berthelot, Cohomologie cristalline des schémas de charactéristique $p>0$, Springer LNM 407, 1974. 
[B-O] P. Berthelot and A. Ogus, Notes on crystalline cohomology, Princeton, 1978.

[Bl] S. Bloch, Crystals and de Rham-Witt connections, J. Inst. Math. Jussieu, 3 (2004), no. 3, 315-326.

[E] J.-Y. Etesse, Complexe de De Rham-Witt à coefficients dans un crystal, Comp. Math., 66 (1988), 57-120.

[H] R. Hartshorne, Residues and Duality, Springer LNM 20, 1966.

[K] N. Katz, Nilpotent connections and the monodromy theorem: Applications of a result of Turritin, IHES Publ. Math. No.3 (1970).

[K-O] N. Katz and T. Oda, On the differentiation of de Rham cohomology classes with respect to parameters, J. Math. Kyoto Univ., 8-2 (1968), 199-213.

[L-Z] A. Langer and Th. Zink, De Rham-Witt cohomology for a proper and smooth morphism, preprint (2001). http://www.mathematik.uni-bielefeld.de/ zink.

Andreas Langer

Mathematics

University of Exeter

Exeter, EX4 $4 Q E$

Devon

$U K$

a. langer@ex.ac.uk

Thomas Zink

Fakultät für Mathematik

Universität Bielefeld

Postfach 100131

D-33501 Bielefeld

Germany

zink@mathematik . uni-bielefeld.de 